\title{
IMPROVED BLOCK STAGEWISE REGULARIZED ORTHOGONAL MATCHING PURSUIT IMAGE RECONSTRUCTION METHOD
}

\author{
Xiong-yong Zhu ${ }^{1}$, Shun-dao Xie ${ }^{2,3}$, Guo-ming Chen $^{1}$, Liang Xue ${ }^{1}$, \\ Wen-fang $\mathrm{Wu}^{2}$, Hong-zhou $\mathrm{Tan}^{2,3 *}$ \\ ${ }^{1}$ Department of Computer Science, \\ Guangdong University of Education Guangzhou, China \\ ${ }^{2}$ School of Electronic and Information Engineering \\ Sun Yat-Sen University Guangzhou, China \\ ${ }^{3}$ SYSU-CMU Shunde International Joint Research Institute, Foshan, China
}

\begin{abstract}
Traditional methods to signal acquisition need to collect large amounts of redundant data, and then compress the data to extract useful information, which is inefficient and requires large amount of storage resources. Compressed sensing (CS) can avoid sampling the redundant data; it obtains the discrete signals at the sampling rate that is lower than the Nyquist sampling rate, and reconstructs the original signal with high probability. Based on CS, Block Stagewise Regularized Orthogonal Matching Pursuit (StROMP) is proposed in this paper to reconstruct images. Simulation results show that the proposed algorithm can effectively reduce the required storage storages and computational complexity, which improves the quality of reconstructed images in the premise of ensuring a shorter reconstruction time.
\end{abstract}

\section{KEYWORDS}

Compressive Sensing; Matching Pursuit; Image Reconstruction

\section{INTRODUCTION}

The traditional method to signal acquisition is based on the theorem of Nyquist sampling. We usually proceed by all the original data and then compress it to get the actionable message. However, most of the data that we acquire can be thrown away with almost no perceptual loss. The classical way wastes a great deal of sampling resources, and requires more resources for data storage and processing. Then compressive sensing emerges as a new sampling theory. Sampling and compression get on simultaneously in CS. In other words, it no longer deals with analog to digital conversion, but analog to information conversion (AIC), which dramatically reduces the sampling rate as well as the cost of data storage and transmission [1]. Efficient reconstruction method is the key in CS. There are three main types [2] as show in the following.

Convex relaxation. These methods only need a small number of observations, but with high computational burdensome and long time to carry out. 
Combinatorial algorithms. These methods can support for rapid reconstruction. Nevertheless, the quality of reconstructed image is poor than that of convex relaxation.

Greedy pursuits. These algorithms are intermediate in their running time and reconstruction quality [3], which have simple structure and low computation. Examples include Matching Pursuit(MP)[4],Orthogonal Matching Pursuit(OMP)[5], regularized Orthogonal Matching Pursuit(ROMP)[6], Stagewise Orthogonal Matching Pursuit(StOMP)[7]. Wu J \& Liu F[8] proposed the Multivariate Pursuit Algorithm(MPA), which is based on the multi-scale model to reconstruct the image in the wavelet domain. But it's dependent on the scale model too much. Budhiraja S [9] improves the OMP algorithm for better performance, but running time is still longer than the StOMP algorithm.

We should realize that the reconstruction time and the quality of reconstructed image are mutually restricted in CS. In addition, when CS is applied to 2D images, the measurement matrix can be fairly large, which makes several challenges including a computationally expensive reconstruction process and huge memory required to store the sampling operator. To solve this problem, Gan L proposed Block Compressed Sensing (BCS)[10]. It only needs to store the random matrix of blocked image that has compact size. And the encoder can transmit each block after its linear projection instead of waiting until the entire image is measured. However, the quality of the reconstructed image declines because BCS does not consider the global sparsity, and neglect the difference of the information distribution between blocks. Therefore, it is still a key problem to find the effective and reasonable method to segment and reconstruct images.

Therefore, this work focuses on how to design efficient image reconstruction method. The aim to attain better balance in the reconstruction time and the reconstruction quality, then we proposed the improved block stage wise regularized orthogonal matching pursuit (BStROMP) method. Simulation shows that the proposed algorithm can support for rapid reconstruction and ensure the quality of the images.

The rest of this paper is organized as follows. Section II provides the mathematical model of image reconstruction based on compressed sensing. Section III presents the proposed BStROMP algorithm. Section IV reports analysis of the simulation results followed by conclusions in Section V.

\section{THE MATHEMATICAL MODEL OF IMAGE RECONSTRUCTION BASED ON CS}

The framework of CS theory includes three aspects, the sparsity of the signal, the design of the measurement matrix and the robustness reconstruction method [1]. The idea of sparse expression is that when using the appropriate orthogonal basis as signal representation, the vast number of natural signals and images can be sparse or compressible[11]. The role of the measurement matrix is to reduce the dimensions thus CS can reduce the number of samples, and the reconstruction matrix needs to be irrelevant to the sparse representation matrix. The reconstruction method means that we can recover the original signal by solving the optimization problem [12]. In Figure

1 , sampling and compressing are at the same time. Sampling M discrete data to avoid redundant samples directly, it reduces the cost of storage and transmission. What's more, the complexity of information acquisition shifts from the sampler to the receiver [13]. 


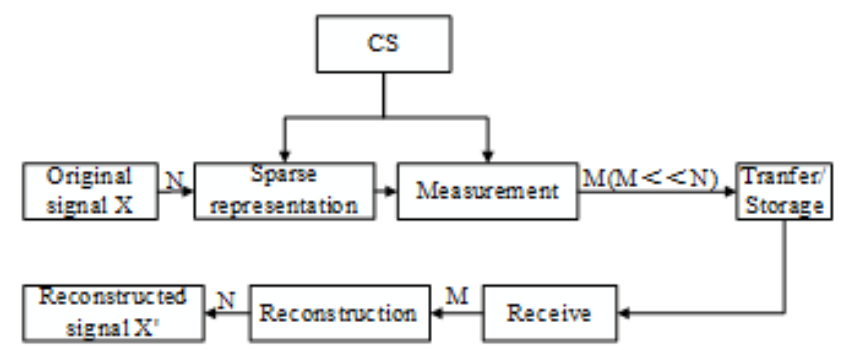

Figure 1. Flow chart of signal reconstruction based on compressive sensing

$x=[x(1), x(2), \ldots x(N)] \in R^{N}$. Sampling $\mathrm{x}$ at the rate much lower than Nyquist rate, the measurement matrix is denoted by $\boldsymbol{\Phi}(M \times N, M<<)$. Then we can get the $M \times 1$ measurement vector $y \in R^{M}$ from equation (1) and (2).

$$
\begin{aligned}
& y=\Phi x \\
& x=\psi \alpha
\end{aligned}
$$

In equation (2), $\psi \in R^{N \times N}$, sparse vector $\alpha \in R^{N}$ is called the K-sparse expression of $\mathrm{x}$ [14] , which means that it contains only K nonzero element [11] . From equation (1) (2), we have

$$
y=\Phi \psi \alpha=\Theta \alpha
$$

Among equation (3), $\Theta=\Phi \psi$, it is called holographic dictionary or library[11]. When $\Theta$ satisfies the condition of RIP, we can obtain the estimation $\mathscr{k}$ of the sparse signal $\alpha$ by equation (3). Then

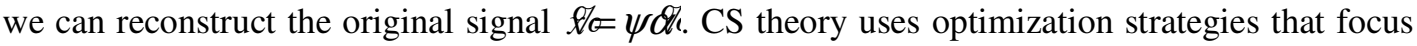
on finding the sparsest signal that matches with the $\mathrm{M}$ projections in y to reconstruct the signal. It is specific outstanding advantages. In other words, the reconstruction problem can be recast as a minimization $l_{0}$ problem.

$$
\min _{\alpha} \mathrm{P} \alpha \mathrm{P}_{0} \text { s.t. } y=\Phi \psi \alpha
$$

Unfortunately, the $l_{0}$ optimization problem is NP-hard. Much work has been done to show that under some specific circumstances, a convex optimization problem can be used to recover such signals. These results show that the sparse recovery problem is equivalent to the $l_{1}$ convex program [11].

$$
\min _{\alpha} \mathrm{P} \alpha \mathrm{P}_{1} \text { s.t. } y=\Phi \psi \alpha
$$

In fact, real signal would always accompany with noise. The equation (5) can convert to the optimization problem in equation (6) [11].

$$
\min _{\alpha} \mathrm{P} \alpha \mathrm{P}_{1} \text { s.t. } \mathrm{P} y-\Phi \psi \alpha \mathrm{P}_{1} \leq \varepsilon
$$


For a positive constant $\mathrm{C}$, if $M \geq C \mu^{2}(\Phi, \psi) K \log (N / \delta)$ holds, the solution $\alpha$ of the $l_{1}$ norm minimization problem can be accurately calculated by the probability of $1-\delta$. Thereby the high-dimensional discrete vector $\mathrm{x}$ can be reconstructed from the low-dimensional sampling vector $\mathrm{y}$ with a probability of $1-\delta[11]$.

\section{THE BSTROMP ALGORITHM}

Based on block CS, different reconstruction methods require different running time, and they would lead to different reconstruction quality. Basic pursuit (BP) [15] is a $1_{1}$ convex program, with high reconstruction accuracy and good reconstructed image quality. However, its running time is the longest among all the method that we mentioned. Compared with BP, the running time of the OMP [5] is reduced, and the quality of reconstructed image is relatively good, but the running time is still long. StOMP [7] reduces its running time dramatically, but the reconstructed image quality is worst. Budhiraja proposed modified orthogonal matching pursuit (MOMP) [16], the quality of reconstructed image improves a lot, but MOMP also need long time to run. Gao X presents Block Sparse Adaptive Regularized Matching Pursuit (BSARMP)[17]. To a certain extent, the running time is short than other methods, but the time is still too long. What's more, it does not have the same performance as BP.

In order to solve the problem above, we improve the StOMP algorithm, and then propose the BStROMP.

\subsection{Block Compressed Sensing of Natural Images}

Suppose $\mathrm{x}$ is a $N_{r} \times N_{c}$ image, We can transfer the 2-D image to 1-D signal, and then sample the 1-D signal to take M CS measurements with lower sample rate, and the original image can be recovered by reconstruction algorithm. However, when the length of signal is too large, the measurement matrix and the storage needed would be too large [10]. Therefore, we segment the image into several blocks as shown in figure 2 .

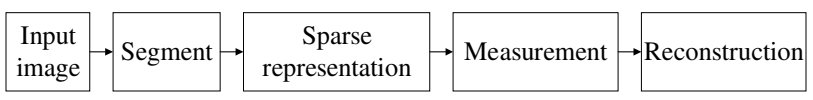

Figure 2. Process of block compressed sensing

The input image with $\mathrm{N}=\mathrm{N}_{\mathrm{r}} \mathrm{N}_{\mathrm{c}}$ pixels in total will be divided into $\mathrm{n}$ small blocks with size of $B \times B$. Let $x_{i}, i=1,2, \ldots x_{n}\left(n=N / B^{2}\right)$ represent the vectorized signal of the $\mathrm{i}$-th block through raster scanning. We use the same matrix $\Phi_{B}$ to sample each block[10]. The corresponding output of CS vector can be expressed as

$$
y_{i}=\Phi_{B} x_{i}, i=1,2, \ldots, n
$$

Where $\Phi_{B}$ is represented as Gaussian matrix with size of $\mathrm{n}_{\mathrm{B}} \times \mathrm{B}^{2}$ generally, in which, $n_{B}=\left\lfloor\frac{M B^{2}}{N}\right\rfloor$, and $B$ can be $8,16,32$ and so on. For the whole image, the equivalent sampling operator $\Phi$ is thus a block diagonal matrix taking the following form [10] 


$$
\Phi=\left|\begin{array}{cccc}
\Phi_{B} & 0 & 0 & 0 \\
0 & \Phi_{B} & 0 & 0 \\
0 & 0 & \Phi_{B} & 0 \\
0 & 0 & 0 & \Phi_{B}
\end{array}\right|
$$

In equation (8), we can figure out that we never need to storage a full $n \times N$ matrix. Instead, Block CS is memory efficient for we just need to store a $n_{B} \times B^{2}$ Gaussian ensemble. In this way, we can reduce the computational complexity. However, if we use the same operator to sample each block, the loss of difference between blocks would cause the quality decline of the reconstructed image. What's more, it may cause block artifacts, ringing effect or distortion in edge detail. Therefore, according to the characteristics of the image, we divide all the image blocks into three types: Smooth, Edge and Texture. According to their different features, we set different weight for the number of measurement times. The image blocks are classified by calculating the variance

$$
\begin{gathered}
v=\frac{1}{B \times B} \sum_{j=1}^{B \times B}\left(\mathrm{p}_{\mathrm{j}}-\overline{\mathrm{p}}\right)^{2}, \overline{\mathrm{p}}=\frac{1}{B \times B} \sum_{j=1}^{B \times B} \mathrm{p}_{\mathrm{j}} \\
v^{\prime}=\frac{v-v_{\min }}{v_{\text {max }}-v_{\min }}
\end{gathered}
$$

Where $v$ is the variance, and $p_{j}$ is the $\mathrm{j}$-th pixel value of the block. $v_{\max }$ and $v_{\min }$ are the maximum and the minimum of the variance in all the image blocks respectively. By equation (10), we can classify $X$.

$$
x_{i}=\left\{\begin{array}{c}
\text { Smooth, if } \quad v^{\prime} \leq T \\
\text { Texture, if } \quad v^{\prime}>T_{2} \\
\text { Edge, } \text { if } T_{1}<v^{\prime} \leq T_{2}
\end{array}\right.
$$

When $v^{\prime} \leqslant 0.1, x_{i}$ is regarded as smooth, and when $v^{\prime} \geqslant 0.3$ is classified as Texture, and the rest is Edge.

Assume that we have $\mathrm{n}$ image blocks in total, we set the sampling rate of smooth, edge, texture to $r_{1}, r_{2}, r_{3}$ respectively. And the number of blocks of each part is $n_{1}, \mathrm{n}_{2}, \mathrm{n}_{3}$ respectively.

Suppose $\mathrm{r}$ is the fixed sampling rate for the whole image, we have

$$
n \times B^{2} \times r=n_{1} \times B^{2} \times r_{1}+n_{2} \times B^{2} \times r_{2}+n_{3} \times B^{2} \times r_{3}
$$

In equation (12), we set $r_{1}=R, r_{2}=3 R, r_{3}=5 R$. Then we have

$$
R=\frac{N \times r}{N_{1}+3 N_{2}+5 N_{3}}
$$


We can calculate $r_{1}, r_{2}$ and $r_{3}$ by $R$. We control the sampling rate of different part through different weight. Higher sampling rates for images containing rich information prevent the loss of details and texture information after refactoring. The lower sampling rate for less information can also lead to good performance.

\subsection{ACGP algorithm}

In StOMP, we calculate the correlation coefficient first and then use a hard threshold to judge the new column to update the support set. In this way, we can speed up the iteration but the support set updated is not optimal in each iteration, which makes the reconstruction accuracy greatly reduced [3]. In order to solve the problem, we use regularization method to verify the threshold. To reduce the complexity of the StOMP algorithm, we adopt an approximate conjugate gradient algorithm to estimate the approximate solution the signal.

Set the equation

$$
A x=b, x \in R^{n}
$$

Where $A \in R^{m \times n}$ is a symmetrical positive determined matrix. We use conjugate gradient algorithm[18] to solve equation (14), it is equivalent to find the minimum of $y$ in equation (15), i.e. to solve a quadratic optimization problem.

$$
y=\frac{1}{2} x^{T} A x-b^{T} x, x \in R^{n}
$$

Assume that $p, q \in R^{n}$, both $\mathrm{p}$ and $\mathrm{q}$ are not zero. If equation (16) holds, then the vectors $\mathrm{p}$ and $\mathrm{q}$ are A orthogonal or conjugate.

$$
p^{T} A q=0
$$

if $\mathrm{d}_{1}, \mathrm{~d}_{2}, \ldots, \mathrm{d}_{\mathrm{n}} \in \mathrm{R}^{\mathrm{n}}$, for $\forall \mathrm{i} \neq \mathrm{j}$ and equation (17) holds, then $D=\left\{d^{i}, d^{2}, \ldots, d^{n}\right\}$ is the conjugate vector group of $\mathrm{A}$.

$$
\left(d^{i}\right)^{T} A d^{j}=0
$$

If the orthogonal matrix $\mathrm{A}$ is a unit matrix, then $\mathrm{p}$ and $\mathrm{q}$ are orthogonal obviously, and we can see that the conjugate is the expansion of orthogonal [11].

For $\forall x^{0} \in R^{n}$, after $\mathrm{n}$ iterations at most, we can get the solution of equation (14) by equation (18).

$$
\hat{x}^{n}=\hat{x}^{n-1}+\alpha^{n} d^{n}
$$

Where $\mathrm{d}$ is an update direction or conjugate. We adopt the approximate Conjugate Gradient Pursuit (ACGP) method for lower computational complexity and less storage space. We don't need all previously chosen directions but the current to calculate the new direction. The progress is as follows.

Without loss of generality, we set $\mathrm{b} 0=1$, and the gram matrix $\mathrm{G}=\Phi^{\mathrm{T}} \Phi$, and $\mathrm{g}^{\mathrm{n}}$ is the product of the measurement matrix and residual in $\mathrm{n}$-th iteration. Let $\mathrm{G}$ conjugate to all the previously chosen directions, then we have 


$$
<G d^{(n-1)}, g^{n}+b_{1} d^{(n-1)}>=0
$$

We can calculate $b_{1}$ from equation (19).

$$
b_{1}=\frac{<\phi d^{n-1}, \Phi \mathrm{g}^{n}>}{\left\|\Phi d^{n-1}\right\|^{2}}
$$

The new update direction is

$$
d^{n}=g+d^{n-1} b_{1}
$$

With step sizes

$$
\alpha^{n}=\frac{<r^{n}, d^{n}>}{<d^{n}, A d^{n}>}
$$

In summary, we can obtain the residual after this iteration

$$
r^{n}=b-A x^{n}=r^{n-1}-\alpha^{n} A d^{n}
$$

\subsection{The framework of BStROMP}

Based on the mathematical model in part 2 and the improved algorithm in 3.1 and 3.2, the framework of this paper is shown in figure.3.

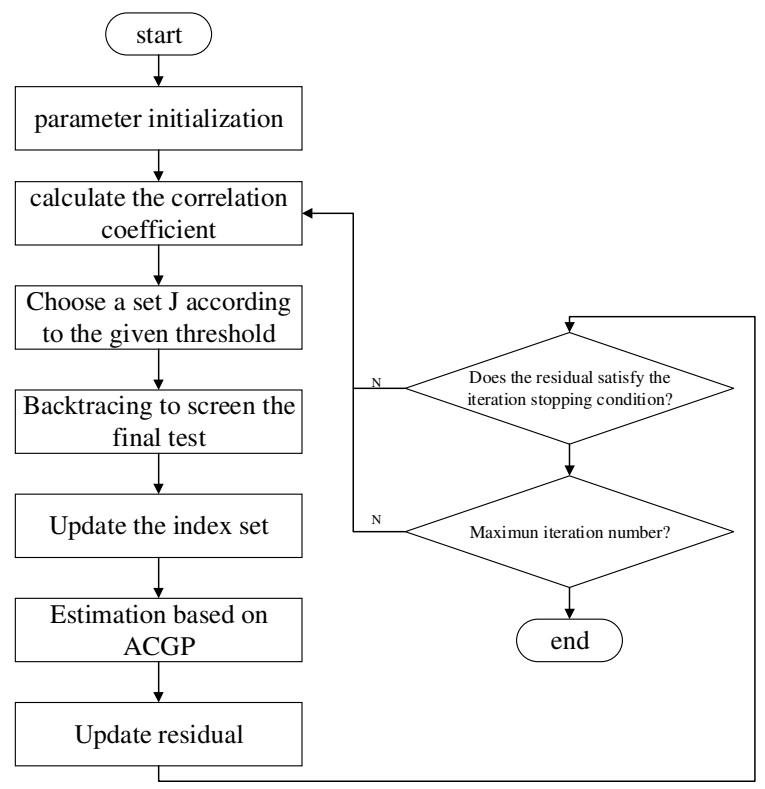

Figure 3. Process of BStROMP

Given the $M \times N$ measurement matrix $\mathrm{A}=\Phi \psi$, and the observation vector $\mathrm{y}$ with size of $\mathrm{M} \times 1$. Through the proposed method in this paper, we can reconstruct the original signal to get the best estimation $\ell$. We will discuss the detail as follows. 
(1) Initialization. Let $\mathrm{r}_{0}=\mathrm{y}, \Lambda_{0}=\varnothing, \mathrm{t}=1$, where $\Lambda_{\mathrm{t}}$ is the index in $\mathrm{t}$-th iteration. $\mathrm{A}_{0}$ is the column vector collection of the matrix A selected by the index.

(2) Calculate. Calculate the correlation coefficient (the absolute value of product) $\mathrm{C}_{t}=\left|\left[A^{T} r_{t-1}\right]\right|$.

(3) Identify. Initial selection $J_{t}=\left\{j: \mathrm{l} \mathrm{c}_{t}(\mathrm{j})>T_{h}=t_{s}\left\|r_{t}\right\|_{2} / \sqrt{M} \mid\right\}$, where $\mathrm{T}_{\mathrm{h}}$ is the hard threshold coefficient.

(4) Secondary choosing. If $\left\|J_{t}\right\|_{0} \neq 0$, then we use regularized principle $|c(i)| \leqslant 2|c(j)|, i, j \in J_{t}$ to choose a set $\left\|u l_{J_{0}}\right\|_{2}$ with maximum energy ; on the contrary, let $\mathrm{J}_{\mathrm{t}}=\max \mathrm{u}_{\mathrm{i}}, \mathrm{i}=1, \ldots, \mathrm{N}$.

(5) Update the support set. $\Lambda_{t}=\Lambda_{t-1} \mathrm{U} J_{t}, A_{t}=A_{t-1} \mathrm{U} \lambda_{j}\left(j \in J_{t}\right), \lambda_{j}$ is the $\mathrm{j}$-th column of $\mathrm{A}$

(6) Calculate $g^{t}=\mathrm{A}_{\Lambda^{t}}^{T} r^{t-1}$. The step size $\alpha_{t}=<r^{t-1}, A_{\Lambda^{t}} d_{\Lambda^{t}}>$, update the new direction $d_{\Lambda^{t}}$.

(7) The estimation of original signal $V_{\Lambda^{t}}=V_{\Lambda^{t}}^{t-1}+\alpha^{t} d_{\Lambda^{t}}$.

(8) Update the residual $r_{t}=y-A_{t} f_{t}$.

(9) $\mathrm{t}=\mathrm{t}+1$. If $\mathrm{t} \leqslant \mathrm{S}$, then go back to (2). If the residual is equal to zero or $\stackrel{\circ}{x}=x_{t}, \mathrm{r}=r_{t}$, then quit the algorithm.

\section{(10) Output $\%$}

The coefficient $t_{s}$ in (3) usually rages from 2 to 3 . The setting of the threshold is very suitable for the reconstruction of Gaussian random signal, but it cannot guarantee the reliability and effectiveness for non-Gaussian signal. When algorithm executes, the atom chosen by the threshold may not meet the conditions, resulting that the support set cannot be optimal in the subsequent cycle. Therefore, we use the regularization to verify the reliability of the threshold.

If the number of atoms selected in step (3) is greater than 0 , the threshold is reasonable, and the selected atoms are regularized to select the optimal set of atoms. If step (3) cannot chose the atom we need, the atom corresponding to the maximum correlation coefficient in the step (2) is chosen. In this case, there is only one atom chosen, and no regularization is necessary.

Step (6) and (7) adopt the ACGP algorithm to solve the estimation of the signal instead of the least squares method. Because of the definite update direction, the speed of iterative convergence is accelerated. Based on the regularization idea above, the performance of BStROMP is improved a lot.

\section{SiMUlationS}

The main criteria to evaluate the performance of CS reconstruction methods are the running time and the reconstructed image quality. Although some reconstruction algorithm have short running time, the quality of the reconstructed image is poor and some of them need long time to run, but the quality of reconstructed image is good. The two criteria restrict each other; we cannot guarantee that the time and the quality are both optimal. We choose Barbara and Baboon in our 
experiments to reconstruct them by BP, OMP, StOMP, BSARMP and proposed algorithm respectively. Then we can compare the running time and the reconstructed image quality of those algorithms. The peak signal-to-noise ratio (PSNR) and reconstruction error are used as objective criteria to evaluate the quality of reconstructed image. In the experiment, the overall sampling rate is $\mathrm{R}=0.5, \mathrm{R}=\mathrm{M} / \mathrm{N}$, where $\mathrm{M}$ is the total number of samples, and $\mathrm{N}$ is the total pixels of the original image. The simulation results are shown below.

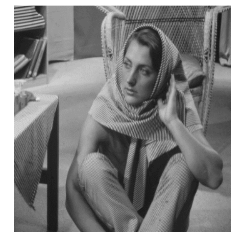

(a) Barbara

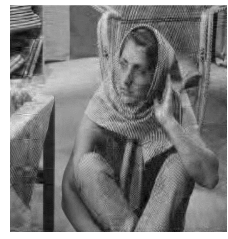

(d) StOMP

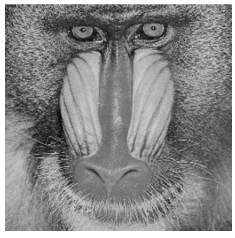

(g) Baboon

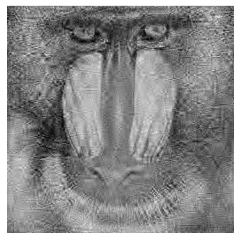

(j) StOMP

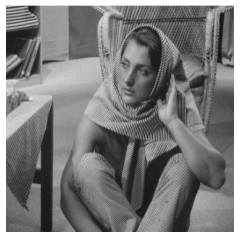

(b) $\mathrm{BP}$

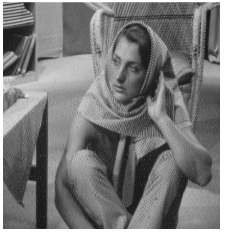

(e) BSARMP

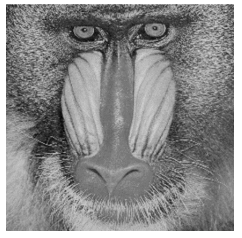

(h) BP

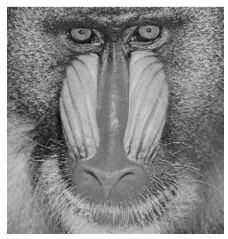

(k) BSARMP

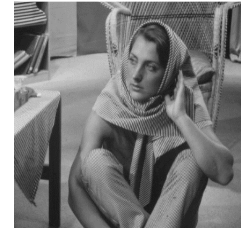

(c) OMP

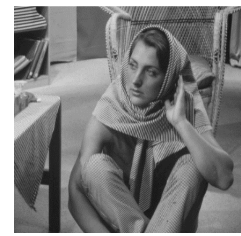

(f) proposed algorithm

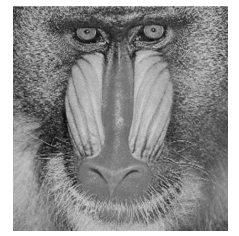

(i) OMP

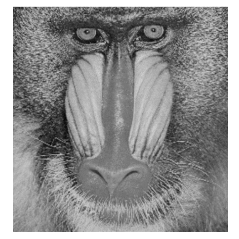

(1) proposed algorithm

Figure 4. Comparison with other CS techniques

Subjectively, according to the analysis of the simulation results of the image above, we can see that the image obtained by the BP algorithm is the best, and the image quality of StOMP is the worst. The performance of proposed algorithm is slightly worse than the BP algorithm, but the difference between all the images above is relatively insignificant from human visual.

To make further compare the performance of each algorithm, we obtain the mean of the evaluation parameters after 50 simulation experiments, as shown in the follows. 
Table 1. Performance comparison of different algorithms

\begin{tabular}{|l|l|l|l|l|l|l|}
\hline \multicolumn{2}{|c|}{ Algorithm } & BP & StOMP & OMP & BSARMP & $\begin{array}{l}\text { proposed } \\
\text { algorithm }\end{array}$ \\
\hline \multirow{2}{*}{ PSNR(dB) } & Barbara & 35.460 & 28.104 & 31.142 & 32.539 & 33.535 \\
\cline { 2 - 7 } & Baboon & 32.383 & 27.818 & 29.738 & 30.106 & 31.379 \\
\hline \multirow{2}{*}{ Time (s) } & Barbara & 15.684. & 0.841 & 8.698 & 2.564 & 1.783 \\
\cline { 2 - 7 } & Baboon & 15.924 & 0.892 & 9.015 & 2.596 & 1.836 \\
\hline $\begin{array}{l}\text { Relative } \\
\text { Error }\end{array}$ & Barbara & 0.0235 & 0.3614 & 0.0483 & 0.0394 & 0.0334 \\
\cline { 2 - 7 } & Baboon & 0.0151 & 0.380 & 0.0492 & 0.0410 & 0.0322 \\
\hline
\end{tabular}

In order to better compare the performance of each algorithm, we also draw the graph of Barbara as follows.

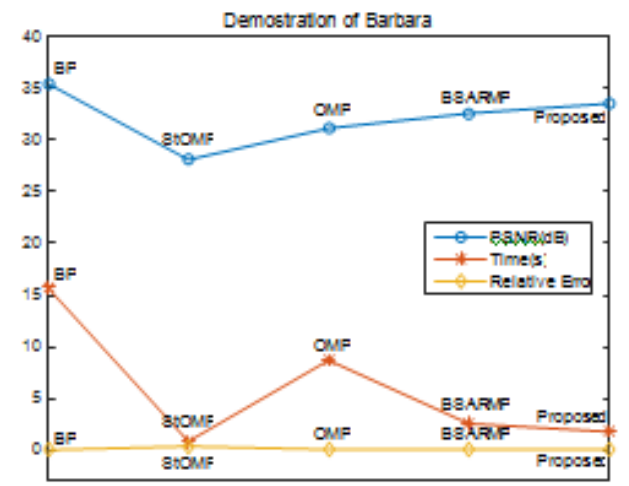

Figure 5. Demostration of Barbara with different CS methods

As seen from table 1 and figure 5, the PSNR of BP is the highest and its quality of reconstructed image is the best when the same sampling rate is used. However, it's running time is the longest among all algorithms. StOMP algorithm has the lowest PSNR, the worst reconstructed image quality, but its running time is the shortest. Although the PSNR and the relative error of the proposed algorithm are lower than BP, compared to other algorithms, the proposed algorithm have better performance, and the running time is much lower than BP, which is only slower than that of StOMP. It can be seen that the time complexity of the proposed algorithm is only higher than that of StOMP, but it is better than BP, OMP and BSARMP. Moreover, the proposed algorithm is improved based on StOMP, with the idea of regularization. Although the reconstruction time is longer than StOMP, the quality of reconstructed image has been greatly improved.

The size of block is also an important factor. We set different size to test the performance of the proposed algorithm: $8 \times 8,16 \times 16,32 \times 32$. Chose Barbara, compare the PSNR in each simulation at different sampling rate. The results are shown in Table 2 .

Table 2. PSNR of different block size

\begin{tabular}{|c|c|c|c|}
\hline $\mathbf{R}$ & $\mathbf{8} \times \mathbf{8}$ & $\mathbf{1 6 \times 1 6}$ & $\mathbf{3 2 \times 3 2}$ \\
\hline 0.1 & $20.034(\mathrm{~dB})$ & $22.612(\mathrm{~dB})$ & $24.069(\mathrm{~dB})$ \\
\hline 0.2 & $22.581(\mathrm{~dB})$ & $24.948(\mathrm{~dB})$ & $27.044(\mathrm{~dB})$ \\
\hline 0.3 & $26.249(\mathrm{~dB})$ & $27.22(\mathrm{~dB})$ & $29.76(\mathrm{~dB})$ \\
\hline 0.4 & $29.452(\mathrm{~dB})$ & $31.156(\mathrm{~dB})$ & $32.630(\mathrm{~dB})$ \\
\hline 0.5 & $31.156(\mathrm{~dB})$ & $32.889(\mathrm{~dB})$ & $33.535(\mathrm{~dB})$ \\
\hline
\end{tabular}


For the typical image such as Lena $(256 \times 256)$, use the image block, and calculate the peak signalto-noise ratio (PSNR) at different sampling rates, the unit is $\mathrm{dB}$, and the experimental results are shown in Figure 6.

As can be seen from table 2, the larger the block image, the better the reconstructed accuracy. However, the storage required for sampling and the computational complexity for reconstruction are also increased. For the representative image such as Lena $(256 \times 256)$, with the block size of $32 \times 32$, the PSNR at different sampling rate is shown in Figure 6 .

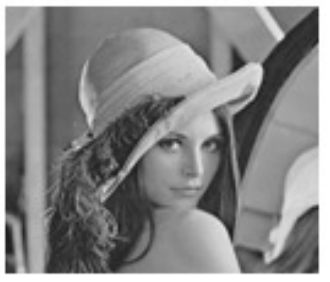

(a) Lena

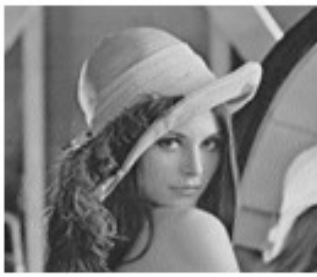

(d) $R=0.3(P S N R=29 d B)$

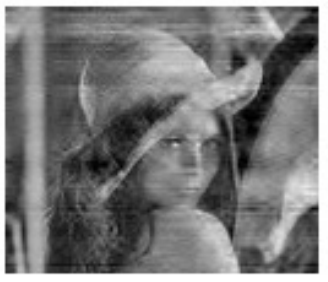

(b) $R=0.1$ (PSNR=25dB)

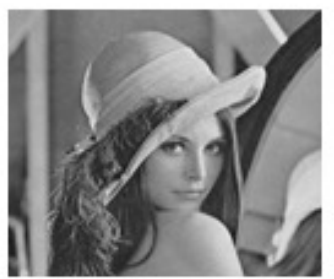

(e) $R=0.4$ (PSNR=32dB)

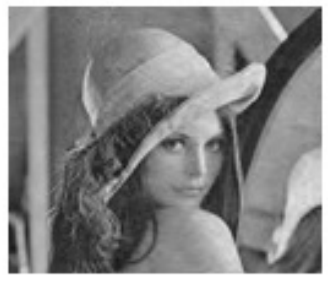

(c) $R=0.2$ (PSNR=27dB)

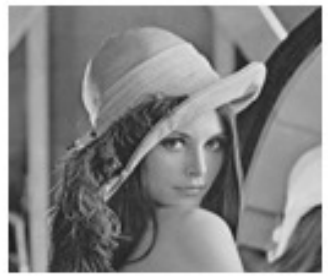

(f) $R=0.5$ (PSNR=35dB)

Figure 6. Demonstration of BStROMP with the same block size $(32 \times 32)$ but different sampling rate

As can be seen from the figure above, with the sampling rate increases, that is, the number of measurements increases, the amount of information obtained as well as the precision would increases. The original image can be reconstructed with high precision when certain conditions are satisfied. Nevertheless, we need to deal with more data at the same time; the complexity and the running time would also increase.

In summary, compared with StOMP, the proposed algorithm improves the reconstructed quality, although the running time is slightly longer than StOMP, which is shorter than other algorithms. The reconstructed quality of the proposed algorithm is inferior compared to BP, but better than OMP and BSARMP.

\section{CONCLUSIONS}

The BStROMP algorithm proposed in this paper reduces the storage and computational complexity required in encoder by using the idea of block CS. Using the atom selection method in StOMP, which is restricted by regularization, improves the precision of reconstructed image. We implement fast reconstruction as well as improving the reconstructed image quality in decoder. The simulation results show the robustness of the proposed algorithm.

However, the proposed algorithm cannot reconstruct the original image well when the sampling rate is extremely low. Therefore, we can further to find a better observation matrix or use other sparse transform to optimize the sparse representation of images. 
The CS reconstruction methods have two problems to resolve, the reconstruction time and the quality of reconstructed image. However, they are contradictory; we cannot guarantee both in the meantime. In addition, the quality of reconstructed image would also affected by environment, so we plan to deal the reconstructed image with post-processing enhancement.

\section{ACKNOWLEDGEMENTS}

This work is supported by National Nature Science Foundation under Grant 61473322 and 61772140, the Technology Planning Project of Guangdong Province under Grant 2017A010101021, the Guangdong Natural Science Foundation - doctoral program under Grant 2016A030310335, and the Foshan science and technology innovation project under Grand 2016AG101823 and 2016AG101793.

\section{REFERENCES}

[1] Shi Guang-Ming, Liu Dan-Hua \& Gao Da-Hua, (2009) "Compressed Sensing Theory and Its Research Progress", Journal of Electronics, Vol. 37, No. 5, pp1070-1081.

[2] Li Shen, Ma Cai-Wen \& Li Yan, (2013)"Review of Compressed Sensing Reconstruction Methods", Infrared and Laser Engineering, Vol. 42, No. S01, pp225-232.

[3] Yang Zhen-zhen, Yang zhen \& Sun Lin-Hui, (2013)"A Review of Orthogonal Matching Pursuit Algorithms for Signal Compression Reconstruction ", Signal Processing, Vol. 29, No. 4, pp486-496.

[4] Mallat S G \& Zhang Z, (1993)"Matching pursuits with time-frequency dictionaries", IEEE Transactions on Signal Processing, Vol. 41, No. 12, pp3397-3415.

[5] Tropp J A \& Gilbert A C, (2007)"Signal recovery from random measurements via orthogonal matching pursuit", IIEEE Transactions on Information Theory, Vol. 53, No. 12, pp4655-4666.

[6] Needell D \& Vershynin R, (2009)"Uniform uncertainty principle and signal recovery via regularized orthogonal matching pursuit", Foundations of computational mathematics, Vol. 9, No. 3, pp317-334.

[7] Donoho D L, Tsaig Y \& Drori I, (2012)"Sparse solution of underdetermined systems of linear equations by stagewise orthogonal matching pursuit", IEEE Transactions on Information Theory, Vol. 58, No. 2, pp1094-1121.

[8] Wu J, Liu F\& Jiao L C, (2011)"Multivariate compressive sensing for image reconstruction in the wavelet domain: using scale mixture models", IEEE Transactions on Image Processing, Vol. 20, No. 12, pp3483-3494.

[9] Budhiraja S, (2015)"Image reconstruction using modified orthogonal matching pursuit and compressive sensing", 2015 IEEE International Conference on Computing, Communication \& Automation (ICCCA), Vol. , No., pp1073-1078.

[10] Gan L, (2007)"Block compressed sensing of natural images", 2007 15th IEEE International Conference on Digital Signal Processing, Vol. , No., pp403-406.

[11] Zhang Xian-Da (2013) Matrix Analysis and Application, Beijing: Tsinghua University Press.

[12] Wenze S \& Zhihui W, (2012)"Advances and perspectives on compressed sensing theory", Journal of Image and Graphics, Vol. 17, No. 1, pp1-12.

[13] Li Zhi-Lin, Chen Hou-Jin \& Yao Chang, (2012)"An Improved Multiscale Compressed Sensing Scheme ", Photoelectron Laser, Vol. 23, No. 7, pp1403-1410.

[14] Candes E \& Romberg J, (2007)" Sparsity and incoherence in compressive sampling", Inverse problems, Vol. 23, No. 3, pp969. 
[15] Chen S S, Donoho D L \& Saunders M A, (2001)"Atomic decomposition by basis pursuit", SIAM review, Vol. 43, No. 1, pp129-159.

[16] Budhiraja S, (2015)"Image reconstruction using modified orthogonal matching pursuit and compressive sensing", 2015 IEEE International Conference on Computing, Communication \& Automation (ICCCA), Vol. , No. , pp1073-1078.

[17] Wang A, Gao X \& Gao Y, (2014)"A Modified Image Reconstruction Algorithm Based on Compressed Sensing", 2014 IEEE Fourth International Conference on Instrumentation and Measurement, Computer, Communication and Control (IMCCC), Vol. , No. , pp624-627.

[18] Hestenes M R \& Stiefel E (1952) Methods of conjugate gradients for solving linear systems, NBS.

\section{AUTHORS}

Xiong-Yong Zhu, Ph.D , Lecturer in the Department of Computer Science, Guangdong University of Education. His research interest covers digital image processing, video signal processing, computer vision.

Shun-Dao Xie, Ph.D student at the School of Electronics and Information Technology, Sun Yat-Sen University. His research interest covers digital image processing, digital integrated circuit design, and Internet of things.

Guo-Min Chen, Received the M.S. and Ph.D. degrees from School of Information Science and Technology, Sun Yat-sen University, China, in 2003 and 2009. He is an associate professor of Guangdong University of Education. His areas of interest include data mining, machine learning, pattern recognition, and image processing.

Wen-Fang Wu, Master student at the School of Electronics and Information Technology, Sun Yat-Sen University. Her main research interest is digital image processing.

Hong-Zhou Tan, Professor at the School of Electronics and Information Technology, Sun Yat-Sen University. His research interest covers broadband communications, signal processing, identification and modeling of complex systems, and semicondector IC design. Corresponding author of this paper.
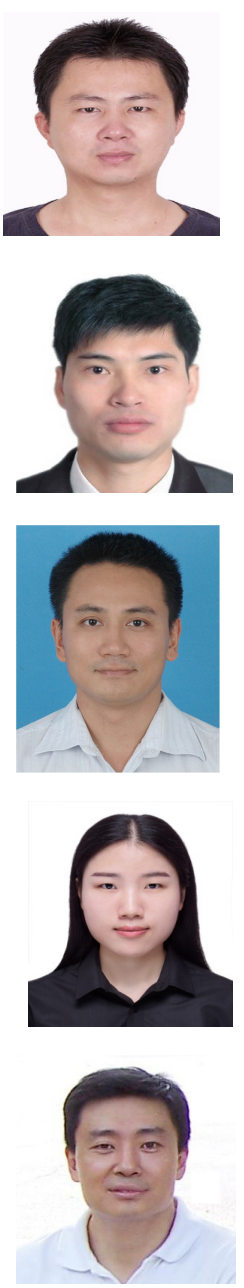